\title{
Frontline Contact Aspiration Treatment for Emergent Large Vessel Occlusion: A Review Focused on Practical Techniques
}

\author{
Dong-Hun Kang, ${ }^{\text {a,b }}$ Yang-Ha Hwang \\ aDepartment of Neurosurgery, Kyungpook National University Hospital, School of Medicine, Kyungpook National University, Daegu, Korea \\ bDepartment of Radiology, Kyungpook National University Hospital, School of Medicine, Kyungpook National University, Daegu, Korea \\ 'Department of Neurology, Kyungpook National University Hospital, School of Medicine, Kyungpook National University, Daegu, Korea
}

\begin{abstract}
Endovascular thrombectomy (EVT) as the standard care for acute stroke due to large vessel occlusion has recently been validated through several randomized controlled trials (RCTs). Contact aspiration (CA) and stent retriever (SR) are the two major EVT methods currently used. Because the RCTs have mostly evaluated SR devices, there was a demand to test CA in relation to SR as a frontline EVT treatment method. Recently, the Contact Aspiration vs Stent Retriever for Successful Recanalization (ASTER) study, the first RCT to compare CA and SR, demonstrated similar efficacy between them as a frontline EVT for patients with large vessel occlusions. This facilitates further investigation to confirm better frontline EVT for patients with acute stroke. In this review, we discuss past and recent developments in CA techniques, focusing on related literature. Additionally, we describe practical skills to overcome technical difficulties that can be encountered during the CA procedure. Finally, we review the evolution of device technologies, including a newer version of using a large-bore aspiration catheter.
\end{abstract}

Correspondence: Yang-Ha Hwang Department of Neurology, Kyungpook National University Hospital, School of Medicine, Kyungpook National University, 130 Dongdeok-ro, Jung-gu, Daegu 41944, Korea Tel: +82-53-420-5758 Fax: +82-53-422-4265 E-mail:yangha.hwang@gmail.com

Received: October 29, 2018

Revised: January 8, 2019

Accepted: January 8, 2019

Keywords Brain infarction; Embolism; Atherosclerosis; Endovascular procedures; Thrombectomy; Reperfusion

\section{Introduction}

The benefit of endovascular thrombectomy (EVT) in the treatment of acute ischemic stroke secondary to large vessel occlusion (LVO) has recently been validated through several randomized controlled trials (RCTs). ${ }^{1-6}$ These trials mostly investigated the latest versions of stent retriever (SR) devices Solitaire (Medtronic, Santa Rosa, CA, USA) and Trevo (Stryker, Mountain View, CA, USA); thus, major stroke guidelines have recommended SRs as a frontline EVT. ${ }^{7-9}$ In practice, another frontline EVT method, contact aspiration (CA), is also used. In a recent survey of United States neurointeventionalists, 39.7\% of respondents re- ported using CA (A Direct Aspiration first Pass Technique [ADAPT]) as a frontline EVT, followed by $28.2 \%$ using SR, while $28.2 \%$ reported a combined usage of SR with $C A .{ }^{10}$ Due to the paucity of studies investigating the effectiveness of $C A$ relative to $S R$, controversy has ensued for several years over which EVT method is superior as a frontline EVT. Recently, the Contact Aspiration vs Stent Retriever for Successful Recanalization (ASTER) study was the first RCT to compare frontline CA versus SR based on patients at 8 French comprehensive stroke centers $(n=381)$. Results demonstrated similar efficacy between SR and CA as a frontline EVT for patients with LVO." Moreover, another RCT, the Comparison of Direct Aspiration vs Stent Retriever as a First Ap- 
proach (COMPASS), has just completed enrollment in the United States $(n=270) .{ }^{12}$ Evidently, the choice of more effective frontline $\mathrm{EVT}$ is one of the most important practical issues, which may be resolved within a couple of years.

There are two key technical elements for achieving successful recanalization during CA: "delivery" of a large-bore aspiration catheter to a thrombus, and "contact" between the tip of the aspiration catheter and proximal surface of the thrombus. With regard to delivery of an EVT device, CA is more technically demanding than SR because of the larger size of the aspiration catheter. ${ }^{13}$ With respect to contact, the optimal location of the tip of the aspiration catheter is crucial for effective clot aspiration. These technical aspects are further described below. In the ASTER trial, authors discussed the importance of doctors' experience in achieving high successful recanalization rates and stated a limitation in generalizing data interpretation to centers with less experience, as all eight participating centers were highly experienced in performing both methods. ${ }^{11}$ In this review, we will briefly discuss the background of CA in the present era of EVT in treatment of acute LVO patients, and then describe in more detail the technical aspects and overcoming difficulties that can be encountered during CA procedures.

\section{Contact aspiration for endovascular stroke treatment: past and present}

Contact or clot aspiration thrombectomy originated from a manual aspiration therapy for large, peripheral vessel occlusions. ${ }^{14}$ In terms of the practical technique, a large-bore catheter from 4 to $8 \mathrm{Fr}$ is advanced to the proximal surface of a clot, then manual aspiration is performed via a syringe. The virtues of this method are that it is fast to perform and technically simple. Nevertheless, the application of the technique was historically restricted to non-tortuous vessels, due to the large and stiff catheters available at the time. ${ }^{15,16}$ However, after the launching the Penumbra System (Penumbra, Alameda, CA, USA), this technique became applicable for acute stroke patients, as the Penumbra reperfusion catheter had a large internal diameter that was more malleable, making it effective at advancing into intracranial arteries. Variations of this newgeneration CA technique are similar in procedure characteristics but differently named: forced aspiration suction thrombectomy (FAST) and ADAPT. ${ }^{17,18}$ The first case series on FAST was published in 2011. ${ }^{17}$ In that case series, 22 consecutive patients with LVO were enrolled with middle cerebral artery (MCA, $n=14)$, internal carotid artery (ICA, $n=4)$, and basilar artery ( $B A$, $n=4$ ) occlusions. After the FAST procedure, $81.9 \%$ of the patients had successfully recanalization, with Thrombolysis In Cerebral Infarction (TICI) Scale 2b/3. In 2013, a new modification of the Penumbra System technique was developed called ADAPT. The main difference between FAST and ADAPT was the generation of Penumbra reperfusion catheters. Thirty-seven consecutive cases were treated with the ADAPT technique, 30 of which involved the anterior circulation. Revascularization was successful in all cases, including $65 \%$ with $\mathrm{TICl} 3$ recanalization. ${ }^{18}$ Over the past few years, new aspiration devices have been developed, including variations in the shaft design and the distal inner diameter, to enhance delivery and aspiration capacity (Table 1). Therefore, data involving CA with new catheters are continuously improving. ${ }^{19-22}$

Table 1. Evolution of large-bore aspiration catheters from the first-generation Penumbra reperfusion catheter

\begin{tabular}{|c|c|c|c|c|c|}
\hline Product name (company) & Length $(\mathrm{cm})$ & Proximal OD (inch) & Proximal ID (inch) & Distal OD (inch) & Distal ID (inch) \\
\hline 054 reperfusion catheter (Penumbra) & 132 & 0.080 & 0.064 & 0.066 & 0.054 \\
\hline 5Max reperfusion catheter (Penumbra) & 132 & 0.080 & 0.064 & 0.065 & 0.054 \\
\hline ACE 64 (Penumbra) & 132 & 0.080 & 0.068 & 0.075 & 0.064 \\
\hline ACE 68 (Penumbra) & 132 & 0.080 & 0.068 & 0.080 & 0.068 \\
\hline JET 7 (Penumbra) & 132 & 0.085 & 0.072 & 0.075 & 0.072 \\
\hline ARC (Medtronic) & 132 & 0.080 & 0.069 & 0.069 & 0.061 \\
\hline React 68 (Medtronic) & 132 & 0.083 & 0.068 & 0.083 & 0.068 \\
\hline Catalyst 6 (Stryker) & 132 & 0.079 & 0.060 & 0.071 & 0.060 \\
\hline Catalyst 7 (Stryker) & $125 / 132$ & 0.0825 & 0.068 & 0.082 & 0.068 \\
\hline Sofia (MicroVention) & 125 & 0.068 & 0.055 & 0.068 & 0.055 \\
\hline Sofia Plus (MicroVention) & $125 / 131$ & 0.083 & 0.070 & 0.082 & 0.070 \\
\hline
\end{tabular}

$\mathrm{OD}$, outer diameter; ID, inner diameter. 
Multicenter Randomized Clinical Trial of Endovascular Treatment for Acute Ischemic Stroke in the Netherlands (MR CLEAN), presented in 2014, was the first RCT to report beneficial results for EVT compared to intravenous thrombolysis in acute stroke due to LVO specifically in the anterior circulation.' This study was followed by five more trials reporting positive results. ${ }^{2-6}$ All of these RCTs reported an increased rate of successful recanalization (modified $\mathrm{TICl} 2 \mathrm{~b} / 3$ ), ranging from 59\% to $88 \%$. More importantly, this technical success translated into clinical improvement, which resulted in favorable clinical outcome (modified Rankin Scale [mRS] 0-2 at 3 months) with EVT in between $33 \%$ and $71 \%$ of cases. ${ }^{23}$ As described earlier, these trials mostly included SR devices; thus, major stroke guidelines have updated to recommend EVT with frontline SR as standard stroke care. In the perspective of $C A$, there was an RCT named The Randomized, Concurrent Controlled Trial to Assess the Penumbra System's Safety and Effectiveness in the Treatment of Acute Stroke (THERAPY) reported in 2016. ${ }^{24}$ This trial attempted to demonstrate the superiority of aspiration thrombectomy after intravenous-alteplase compared with intravenous-alteplase alone in patients with LVO, but failed to achieve the primary endpoint, showing similar rates of functional recovery in both treatment groups (mRS 0-2 at 90 days, EVT 38\% vs. intravenous-alteplase 30\%, $P=0.52$ ). However, interpretation of this data requires caution as THERAPY was halted early after 108 patients (of 692 originally planned), due to external evidence from the aforementioned SR-based RCTs. Moreover, THERAPY reported $73 \%$ successful reperfusion (modified $\mathrm{TICl} 2 \mathrm{~b} / 3$ ) in a $\geq 8$-mm clot burden population, which was consistent with the other RCTs ranging from 59\% (MR CLEAN) to 88\% (Solitaire with the Intention for Thrombectomy as Primary Endovascular Treatment [SWIFT-PRIME]), and improvements in all additional secondary endpoints including mRS at 30 days, National Institutes of Health Stroke Scale, and infarct volume using the Alberta Stroke Program Early CT Score at 24 hours, demonstrated consistent directions of a beneficial effect of EVT. ${ }^{23,24}$

Although the results of THERAPY were somewhat disappointing, there was no reduction in the use of CA due to the obvious potential role of CA as a rescue of SR-based EVT. ${ }^{10} \mathrm{SR}$ is not always successful, as demonstrated by the Highly Effective Reperfusion evaluated in Multiple Endovascular Stroke Trials (HERMES) collaboration study that pooled individual data of the five RCTs and showed that 29\% of patients in the EVT arm failed to achieve successful recanalization. ${ }^{23}$ Furthermore, there have been several attempts to improve results of frontline $C A$ by using switching strategy from $C A$ to $S R \cdot{ }^{25,26} A$ comparison of data from 119 patients treated with frontline SR
(Solitaire FR, Medtronic) and 124 with ADAPT using Penumbra reperfusion catheters was reported. ${ }^{25}$ The authors actively utilized the advantage of ADAPT in the case of failure of the frontline ADAPT approach, by using the large-bore aspiration catheter as a conduit for introducing a SR or another adjunctive device. As a result, frontline CA in the ADAPT group yielded a higher rate of successful revascularization at final angiography than frontline SR ( $82.3 \%$ vs. $68.9 \%, P=0.022)$; however, the use of adjunctive devices was higher in the ADAPT group than in the Solitaire group (ADAPT 38.7\% vs. Solitaire 13.3\%, $P<0.001)$. Although the rate of successful recanalization by ADAPT alone was only $50.8 \%$ (63/124), rescue switching to SR resulted in successful recanalization in 69.6\% (39/56) of cases. Thus, they concluded the benefit of the frontline ADAPT strategy is largely derived from a higher percentage of rescue therapy. Interestingly, despite the markedly higher rate of adjunctive device use in the ADAPT group, it did not lead to a prolonged procedure time (ADAPT 45 minutes vs. Solitaire 50 minutes, $P=0.42$ ). Although such a difference did not result in better clinical outcomes (mRS 0-2 at 3 months, ADAPT 53\% vs. Solitaire $54.8 \%, P=0.79$ ), this necessitated further RCTs to define a better frontline EVT strategy. The "switching concept" was first reported with a period-to-period comparison analysis. ${ }^{27}$ In that study, a CA-only strategy for recanalization was used in period 1 , while a "switching strategy" (from CA to SR in cases of CA failure after three attempts) was used in period 2. It was hypothesized that additional attempts with a different mechanism of SR using Solitaire could improve recanalization in difficult CA cases. As a result, patients in period 2 showed a trend for higher $\mathrm{TICl} 2 \mathrm{~b} / 3$ recanalization (73.8\% vs. $85.1 \%, P=0.10$ ) and significantly better functional outcome at 3 months (49.2\% vs. $67.6 \%, P=0.03$ ). More recently, another study compared frontline CA $(n=47)$ and SR $(n=70)$ strategies. ${ }^{26}$ The authors emphasized the importance of early, protocolized switching to rescue SR for achieving faster and better recanalization. They typically attempted a single pass with the aspiration catheter, and, if adequate recanalization was not achieved, deployed a SR through the aspiration system in order to use CA and SR simultaneously (Combined ASPiration and stentrievER [CASPER]) on the next attempt. In the frontline CA group, the rate of successful reperfusion ( $\mathrm{TICl} 2 \mathrm{~b} / 3$ ) with a single pass of ADAPT was $57 \%$, which resulted in a mean number of 1.3 ADAPT attempts, 20 (42.5\%) of which required rescue SR. Procedure time ( 54.0 minutes vs. 77.1 minutes, $P<0.01$ ) and time to a $\mathrm{TICl} 2 \mathrm{~b} / 3$ recanalization (294.3 minutes vs. 346.7 minutes, $P<0.01)$ were significantly lower in the frontline $C A$ group compared to frontline SR. However, the rates of final $\mathrm{TICl} 2 \mathrm{~b} / 3$ recanalization ( $82.9 \%$ vs. $71.4 \%, P=0.19)$ and good functional 
outcome at 90 days ( $48.9 \%$ vs. $41.4 \%, P=0.45)$ were similar between the two groups. They concluded that a frontline EVT using CA may be warranted prior to $S R$, based on the higher rate of single pass $\mathrm{TICl} 2 \mathrm{~b} / 3$ reperfusion and early, protocolized switching to rescue SR. The positive effect of single pass $\mathrm{TICl}$ $2 b / 3$ recanalization is consistent with a new concept of "first pass effect. ${ }^{\text {"28 }}$ Although this study used the North American Solitaire Acute Stroke Registry database, which is based on LVO patients treated with only Solitaire FR, they delivered a simple and strong message to contemporary stroke physicians. "First pass effect," which was defined as achieving complete recanalization with a single thrombectomy device pass, was achieved in 89 out of $354(25.1 \%)$ and was associated with significantly higher rates of good clinical outcome. Considering that the $57 \%$ of successful reperfusion with a single pass of ADAPT in the aforementioned study, ${ }_{1}^{26}$ the "first pass effect" should be considered an important parameter in future investigations to define a superior frontline EVT strategy.

In 2017, the ASTER trial, which was the first RCT comparing $\mathrm{SR}$ and $C A$, demonstrated a similar efficacy between the two techniques as a frontline EVT for patients with an LVO. ${ }^{11}$ Of note, the neurointerventionalists employed the assigned technique for at least three attempts prior to switching to rescue therapy, and the primary outcome was the percentage of modified $\mathrm{TICl} 2 \mathrm{~b} / 3$ at the end of an EVT procedure. There was no significant difference in revascularization rates (CA $85.4 \%$ vs. SR $83.1 \%, P=0.53$ ) and the rates of good functional recovery (mRS 0-2 at 3 months, CA $45.3 \%$ vs. SR 50\%, $P=0.38$ ). Although the differences were not statistically significant, rescue therapy was more common in the CA cohort than in the SR cohort (32\% vs. 23.8\%; odds ratio, 1.57; $P=0.05$ ); however, median revascularization times were shorter in the CA cohort (38 minutes vs. 45 minutes, $P=0.10$ ). These results were in concordance with those of two previous retrospective studies, suggesting that $C A$ is a good frontline option if the attending neurointerventionalists can achieve quality reperfusion within a few attempts, and in case of failure, promptly switch to rescue SR per their own protocol. ${ }^{11}$ When performing $\mathrm{CA}$, faster and safer delivery of a large-bore aspiration catheter to a thrombus is a key element in achieving prompt reperfusion. Although the trackability of catheters has extensively evolved, it remains crucial for neurointerventionalists to understand practical techniques to overcome difficulties during delivery of a large-bore catheter. These technical aspects will be further discussed in the following section. Moreover, given that frontline CA strategy commonly requires switching to rescue therapy for achieving successful recanalization, it is important to determine the main causes of switching, such as advancement difficulty, large or hard thrombi, non-embolic etiology including in situ thrombotic occlusions, and tandem occlusion during frontline CA relative to SR. In addition, as the reported limit of frontline EVT attempts in previous studies ranged from 1 to 3 passes, $^{11,25-27}$ determining the appropriate number of CA passes before switching is necessary. As previously described, an RCT comparing SR and CA as a frontline EVT (COMPASS trial) has just completed enrollment in the United States. ${ }^{12}$ Therefore, a metaanalysis of the ASTER and the COMPASS trials will provide further information.

The details of procedural and clinical outcomes with frontline CA techniques are summarized in Table 2.

\section{Technical aspects of contact aspiration}

The following section describes practical techniques of the CA procedure. We have endeavored to describe these as objectively as possible, by reviewing relevant articles. However, this description is inevitably based on the authors' accumulated experience considering the center's long history of frontline CA ( $>700$ cases over 9 years) for the treatment of emergent LVO patients.

\section{Stepwise description of the contact aspiration} procedure, focusing on technical tips and current controversies

The CA procedure is initiated by advancing the guiding catheter to the proximity of the occluded target vessel. There are two common selections for a guiding catheter including an 8 or $9 \mathrm{Fr}$ balloon guiding catheter (BGC) or a $6 \mathrm{Fr}$ neurosheath. Specifically, CA users commonly use a 6 Fr neurosheath (usualIy a Neuron 088 Max, Penumbra), while others use a BGC. Investigations using in vitro and animal stroke models have suggested that flow arrest using a BGC reduces the risk of distal embolization and increases the flow reversal effect compared to a conventional guide catheter..$^{29,30}$ Recent clinical studies also demonstrated that BGC use was associated with a higher rate of successful recanalization including a "first pass effect" and a shorter procedure time in SR-based EVT. ${ }^{28,31,32}$ Therefore, BGC use has been generally recommended in SR thrombectomy. However, there has been a lack of studies determining the role of a BGC in CA for acute LVO patients. Very recently, a comparative analysis was performed between BGC utilization and non-utilization groups in cases of frontline $C A$ cases from a Korean multi-center registry. ${ }^{33} \mathrm{~A}$ total of 429 patients enrolled, and a BGC was used in $45.2 \%$ of patients. Compared to the non-BGC group, $B G C$ utilization significantly reduced the number of $C A$ passes (mean, 2.6 vs. 3.4, $P<0.001$ ), puncture-torecanalization time (mean, 56 minutes vs. 64 minutes, $P=0.018)$, and embolization to distal or different site ( $0.5 \%$ vs. 
$3.4 \%, P=0.045)$. The BGC group showed significantly higher final ( $89.2 \%$ vs. $72.8 \%, P<0.001)$ and first-pass recanalization success rates $(24.2 \%$ vs. $8.1 \%, P<0.001)$. Those results were concurrent with previous clinical studies that involved frontline $S R$, which could be explained by the aforementioned advantages of BGC including a decrease in distal embolization and an increase in the flow reversal effect. The authors agreed with recent suggestions of an additional positive effect of BGC use in EVT. ${ }^{34-36}$ Theoretically, there are two key factors that determine how much force is required to retrieve the clot: the impaction force and the combined force induced from friction and adhesion between the thrombus and the vessel wall. The impaction force is the pressure gradient across the thrombus, which is determined by proximal systemic blood pressure and

Table 2. Summary of procedural and clinical outcomes with frontline contact aspiration techniques

\begin{tabular}{|c|c|c|c|c|c|c|}
\hline Study & No. & $\begin{array}{c}\text { Target } \\
\text { occlusion }\end{array}$ & $\begin{array}{l}\text { Procedure time } \\
\text { (min, mean) }\end{array}$ & $\begin{array}{l}\text { Frontline mTICI } \\
2 b-3 \text { reperfusion } \\
\text { (\%) }\end{array}$ & $\begin{array}{c}\text { Final } m T I C I 2 b-3 \\
\text { reperfusion (\%) }\end{array}$ & $\begin{array}{c}\text { Favorable clinical } \\
\text { outcome }(\%)\end{array}$ \\
\hline \multicolumn{7}{|l|}{ Observational studies: contact aspiration } \\
\hline Kang et al. (2011) $)^{17}$ & 22 & $A C, P C$ & 40.2 & NA & $81.9^{*}$ & 45.5 \\
\hline Hwang et al. (2013) ${ }^{19}$ & 20 & ICA & 62.7 & NA & $64.7^{*}$ & 45 \\
\hline Turk et al. (2014) $)^{18}$ & 37 & $A C, P C$ & $28.1(\mathrm{TICl} 2 \mathrm{~b})$ & NA & $97.3^{*}$ & NA \\
\hline ADAPT FAST (2014) $)^{20}$ & 100 & $A C, P C$ & 36.6 & $78^{*}$ & $95^{*}$ & 40 \\
\hline Eom et al. $(2014)^{56}$ & 32 & $\mathrm{BA}$ & 75.5 & NA & 88 & 34 \\
\hline Vargas et al. (2017) $)^{21}$ & 191 & $A C, P C$ & 37.3 & $74^{*}$ & $94^{*}$ & 54.1 \\
\hline Möhlenbruch et al. $(2017)^{42}$ & 85 & $A C, P C$ & $\begin{array}{c}21^{+} \text {(CA only) } \\
53^{+} \text {(rescue SR) }\end{array}$ & $64.7^{*}$ & $96.5^{*}$ & 49.4 \\
\hline Gory et al. (2018) & 20 & $A C, P C$ & $31^{+}$ & NA & 80 & 35 \\
\hline Sallustio et al. $(2018)^{44}$ & 107 & $A C, P C$ & $40^{+}$ & NA & $84.1^{*}$ & 47.6 \\
\hline \multicolumn{7}{|l|}{ Randomized controlled trials: contact aspiration } \\
\hline THERAPY $(2016)^{24}$ & 55 & $\mathrm{AC}$ & NA & 70 & 73 & 38 \\
\hline ASTER $(2017)^{11}:$ CA & 192 & $A C$ & $38^{+}$ & 63.0 & 85.4 & 45.3 \\
\hline ASTER $(2017)^{11}:$ SR & 189 & $\mathrm{AC}$ & $45^{+}$ & 67.7 & 83.1 & 50.0 \\
\hline \multicolumn{7}{|c|}{ Observational studies: frontline SR vs. CA including switching } \\
\hline Kang et al. $(2013)^{27}:$ CA & 61 & $\mathrm{AC}$ & $60^{+}$ & NA & $73.8^{*}$ & 49.2 \\
\hline Kang et al. $(2013)^{27}$ : SR switching & 74 & $\mathrm{AC}$ & $68^{+}$ & $55.7^{*}$ & $85.1^{*}$ & 67.6 \\
\hline Son et al. $(2016)^{54}:$ CA & 18 & $\mathrm{BA}$ & 62.3 & NA & $100^{*}$ & 44.4 \\
\hline Son et al. $(2016)^{54}$ : SR & 13 & BA & 101.9 & NA & $84.6^{*}$ & 38.5 \\
\hline Delgado Almandoz et al. (2016) ${ }^{22}$ : CA & 45 & $A C$ & 50 & $69^{*}$ & $89^{*}$ & 56 \\
\hline Delgado Almandoz et al. (2016) ${ }^{22}$ : Solumbra & 55 & $A C$ & 51 & $82^{*}$ & $84^{*}$ & 31 \\
\hline Lapergue et al. $(2016)^{25}$ : CA & 124 & $A C$ & $45^{+}$ & 50.8 & 82.3 & 53.0 \\
\hline Lapergue et al. (2016) ${ }^{25}$ : SR & 119 & $A C$ & $50^{+}$ & NA & 68.9 & 54.8 \\
\hline Gory et al. $(2018)^{57}: C A$ & 46 & $\mathrm{BA}$ & $45^{+}$ & NA & 87.0 & 40.0 \\
\hline Gory et al. $(2018)^{57}:$ RR & 54 & $\mathrm{BA}$ & $56^{+}$ & NA & 72.2 & 34.0 \\
\hline Stapleton et al. $(2018)^{26}:$ CA & 47 & $A C$ & 54.0 & 46.8 & 83.0 & 48.9 \\
\hline Stapleton et al. $(2018)^{26}:$ SR & 70 & $A C$ & 77.1 & NA & 71.4 & 41.4 \\
\hline Kang et al. $(2018)^{60}: C A$ & 67 & BA & $44^{+}$ & NA & 94.0 & 40.3 \\
\hline Kang et al. $(2018)^{60}:$ SR & 145 & $\mathrm{BA}$ & $38^{+}$ & NA & 90.3 & 46.9 \\
\hline
\end{tabular}

mTICl, modified Thrombolysis In Cerebral Infarction; AC, anterior circulation; PC, posterior circulation; NA, not available; ICA, internal carotid artery; ADAPT FAST, A Direct Aspiration first Pass Technique forced aspiration suction thrombectomy; BA, basilar artery; CA, contact aspiration; SR, stent retriever; THERAPY, The Randomized, Concurrent Controlled Trial to Assess the Penumbra System's Safety and Effectiveness in the Treatment of Acute Stroke; ASTER, the Contact Aspiration vs Stent Retriever for Successful Recanalization; Solumbra, Solitatire plus Penumbra.

${ }^{*}$ Original $\mathrm{TICl} ;{ }^{\dagger}$ Median value. 
distal retrograde collateral flow. Thus, inflation of a BGC can markedly reduce the systemic blood pressure on the proximal clot surface, resulting in a decrease in the pressure gradient across the clot (Figure 1). ${ }^{36}$ This can enhance the aspiration capacity in CA and SR thrombectomy. However, whether to use a BGC during the CA procedure remains a matter of controversy due to the limited evidence so far. Nonetheless, the positive re-

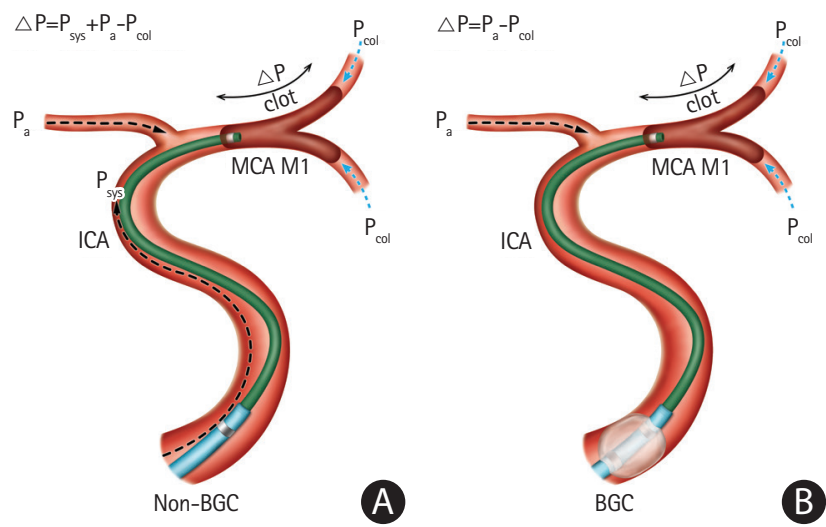

Figure 1. Comparison of the pressure gradient $(\Delta P)$ across the clot between non-balloon guide catheters and balloon guide catheters in contact aspiration procedures. The concept of this figure originated from Yoo et al. ${ }^{36}(A)$ Contact aspiration under non-balloon guide catheter $\left(\triangle P=P_{s y s}+P_{a}-\right.$ $\left.P_{c o l}\right)$. (B) Contact aspiration under balloon inflation of balloon guide catheter $\left(\triangle \mathrm{P}=\mathrm{P}_{\mathrm{a}}-\mathrm{P}_{\text {col }}\right) . \mathrm{MCA}$, middle cerebral artery; ICA, internal carotid artery; $B G C$, balloon guiding catheter. sults of this study warrant a further prospective study in a large population to compare the outcomes of BGC utilization and non-utilization in frontline CA-based EVT.

Once a guiding catheter is optimally positioned, delivery of a large-bore aspiration catheter follows. This is a crucial step for achieving early and quality reperfusion in a CA procedure, because the delivery of an aspiration catheter to the occluded thrombus is not always easy due to the large caliber. ${ }^{13,34}$ Despite recent advances in aspiration catheters' trackability, tortuosity itself and underlying intracranial atherosclerotic stenosis (ICAS) remain obstacles for passage. Moreover, the anatomical position of where an ophthalmic artery originates from the carotid siphon can present an additional challenge. Technical tips to overcome these problems are as follows. Steam shaping of the tip of the large-bore aspiration catheter (Figure 2): if some curves are provided to the tip of the catheter by steamshaping, it theoretically assists in passing the tortuous segment during the FAST procedure. In most cases, delivery is more smoothly achieved by steam-shaping the tip of the catheter to a $45^{\circ}$ curve. However, in some cases with extreme tortuosity or severe underlying ICAS, a $90^{\circ}$ curve or J-shape may be required. Secondly, coaxial advancement technique (Figure 2) can overcome technical difficulty. If a microguidewire alone is used for advancing the large-bore aspiration catheter, the gap between them will be large. This can result in difficulty steering
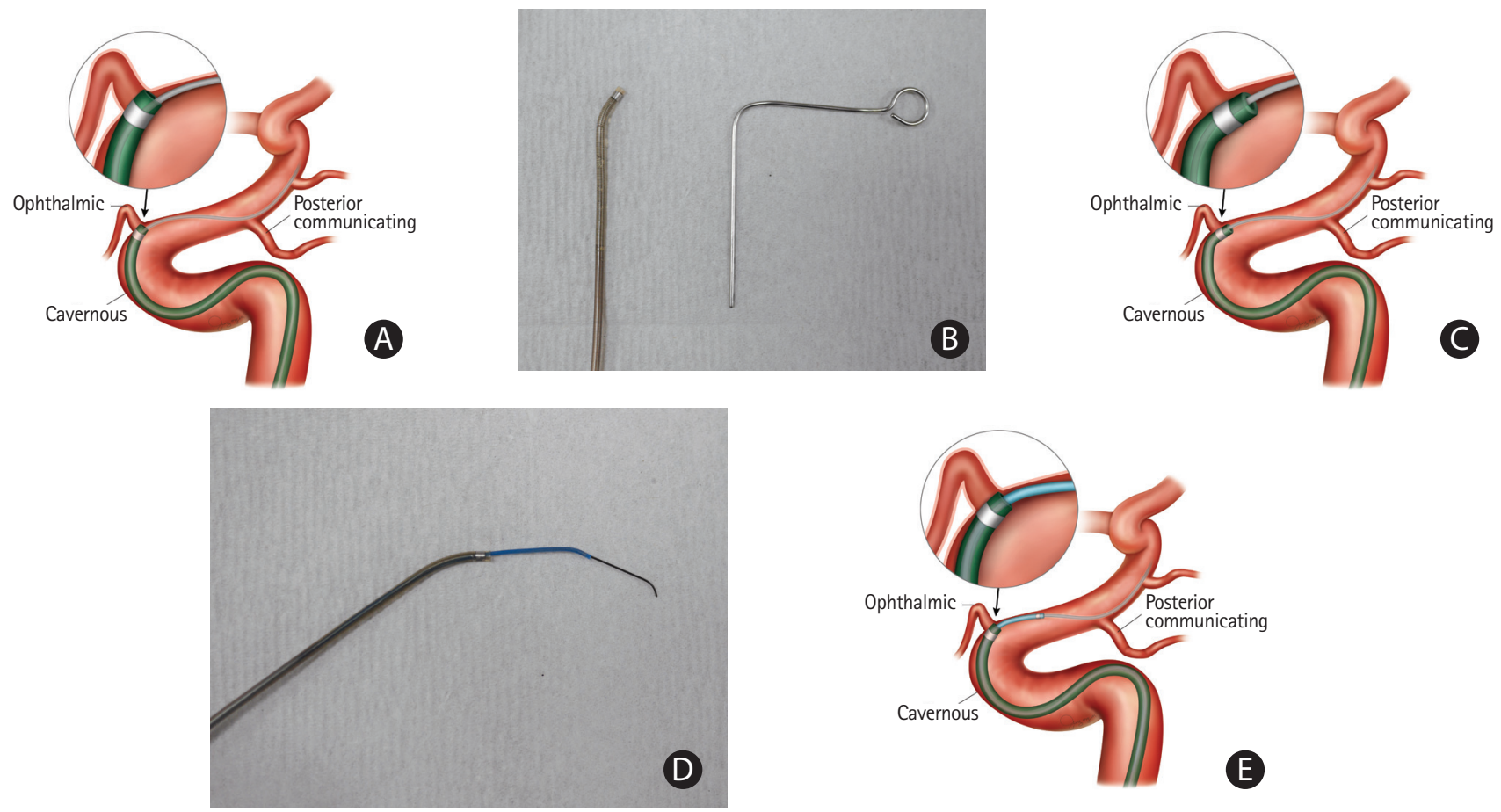

Figure 2. Examples of two technical tips to overcome difficulties during advancement of large-bore catheters. (A) Advancement of a larger catheter through carotid siphon can be challenging during the procedure. Steam shaping of the catheter tip with a $45^{\circ}$ curve $(B, C)$ and the coaxial advancement technique (D, E) may facilitate advancement of aspiration catheter. 
and controlling the catheter, making advancement through a highly tortuous segment unlikely. The coaxial advancement technique is recommended in this situation. Introducing another microcatheter of intermediate size between the aspiration catheter and microguidewire reduces the gap between them, and ultimately allows greater ability to steer and control during advancement. The authors' technical preference for coaxial assembly is as follows. For the ICA and the proximal M1 segment of the MCA, it is recommended to use the ACE 068 (Penumbra) or the Sofia plus (MicroVention, Aliso Viejo, CA, USA) for aspiration with a $2.3 \mathrm{Fr}$ inner microcatheter and a 0.016-inch microguidewire. For smaller arteries, such as the distal M1 segment or the M2 segment of the MCA or BA, the Sofia 5 (MicroVention) or the Penumbra 4 Max for aspiration with a $2.0 \mathrm{Fr}$ inner microcatheter and a 0.014-inch microguidewire are recommended. This combination can be modified according to preference. During this stage, it is recommended to avoid distal passage of the thrombus by a microcatheter or a microguidewire in cases where the patient's vascular anatomy allows, because distal passage itself may theoretically elevate the probability of clot disruption and migration. However, in cases where a patient's arterial anatomy is tortuous, an inner microcatheter is necessarily advanced past the thrombus over a microguidewire. Subsequently, the aspiration catheter is advanced over it until it reaches the thrombus. Local angiography can be performed before aspiration to predict the original path of the occluded segment and to outline the occlusion.

The final step of CA is aspiration of the clot. In this stage, there are two important technical considerations: (1) where to aspirate the thrombus, which involves optimal placement of the tip of the catheter into the thrombus and (2) how to aspirate the thrombus, which means choosing between manual syringe or pump aspiration techniques. For optimal location of the catheter tip placement prior to aspiration, we recommend the catheter tip is advanced into the clot as much as 1 radiopaque marker depth inside (Figure 3). If proper interfacing or contact is achieved, no free blood flow into the tubing is noted. If too proximally located, the tip may easily permit blood flow during aspiration before proper engagement is achieved between the tip and the thrombus; if too distally located, the tip may increase the chance of distal clot migration. When failure occurs with the first attempt of CA, we typically advance the tip 1 more marker depth into the thrombus for better contact. If recanalization is not successful despite a couple of attempts of CA as described, timely switching to rescue SR or CASPER is recommended to reduce procedure time and increase recanalization success. ${ }^{11,25-27}$ The aspiration of the thrombus using negative pressure is the final step of CA. This can be produced by a pump or a manual syringe. Although most ADAPT users employ a pump, controversy remains regarding the benefit of pump usage compared to manual syringe aspiration. ${ }^{37-40}$

\section{Evolution of large-bore aspiration catheters for contact aspiration}

The efficacy of CA is strongly associated with the force of aspiration at the tip of the large-bore aspiration catheter. Therefore, differences in the properties of each catheter may inevitably dictate varying aspiration forces, and thus the efficacy of the procedure. In 2014, one in vitro study compared various parameters of four large-bore catheters commercially
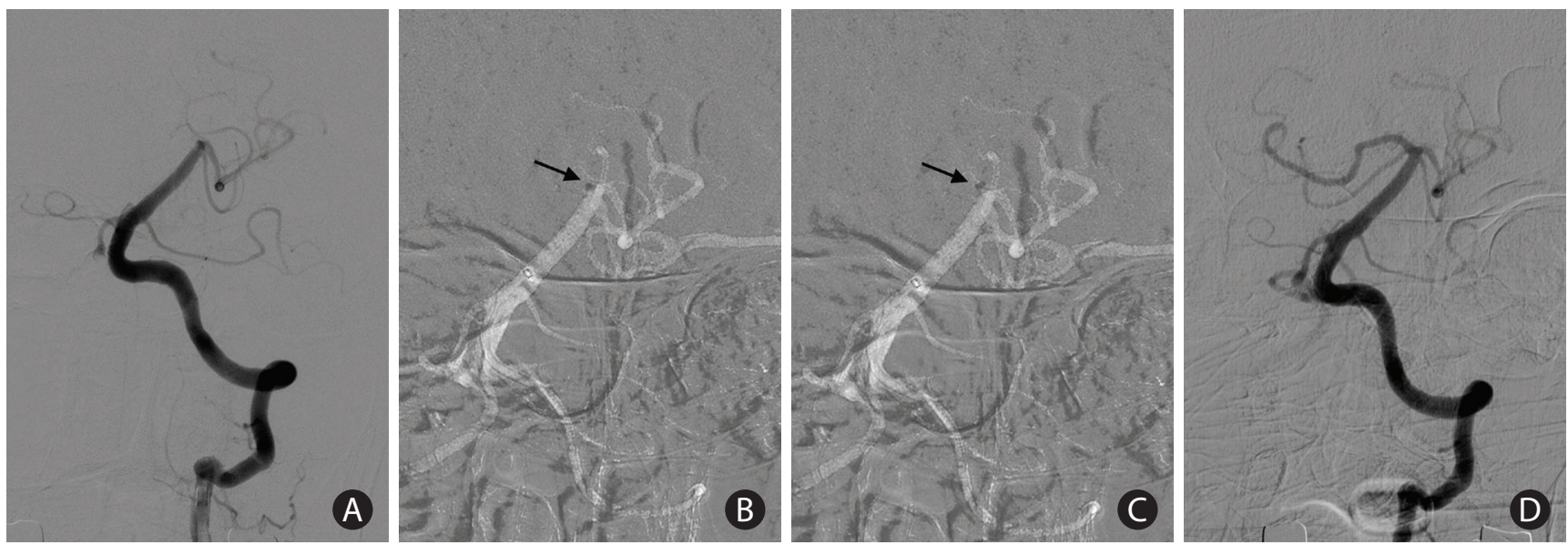

Figure 3. A case showing optimal location of the tip of a large-bore catheter during contact aspiration. (A) A 72-year-old woman presenting with mental changes demonstrated acute occlusion at the top of the basilar artery to right proximal posterior cerebral artery in baseline angiography. (B) During the first attempt, the catheter tip was advanced into the clot as much as 1 radiopaque marker depth inside (arrow). (C) The first attempt failed to retrieve the clot, and the tip was advanced 1 more radiopaque marker depth inside the thrombus for better contact (arrow). (D) Complete recanalization was achieved in the following angiography. 
available at the time. ${ }^{41}$ They analyzed the hemodynamic properties such as catheter tip force, aspiration flow rate, and effective flow lumen to determine the optimal catheter for CA. The study demonstrated that the Penumbra 5 Max ACE outperformed the other catheters in terms of hemodynamic properties, perhaps due to its larger lumen and tapered design. Thus, the mainstay of aspiration catheters for CA became the Penumbra catheter family, which evolved from the first-generation Penumbra reperfusion catheter up to the latest version, JET 7. Recently, several new large-bore catheters have been released for the purpose of aspiration and simultaneous distal access, with a trend for larger internal diameter and softer delivery, including Arc and React 68 by Medtronic, Catalyst 6 and 7 by Stryker, and Sofia and Sofia plus by MicroVention (Table 1). The reported evidence is limited regarding the efficacy of these new catheters compared to Penumbra catheters, as most literature regarding $C A$, including ADAPT and FAST, have involved Penumbra catheters. However, the rates of successful recanalization (modified $\mathrm{TICl} 2 \mathrm{~b} / 3$ ) using the new large-bore catheters were comparable to those of previous reports using Penumbra catheters, which ranged from $64.7 \%$ to $76.6 \%$ with CA alone and $80.0 \%$ to $96.5 \%$ once rescue therapy was applied. ${ }^{42-44}$ Therefore, another in vitro study to compare the hemodynamic parameters of the new catheters relative to Penumbra catheters is warranted. More importantly, the accumulation of experience and clinical data from real practice is required for the thorough evaluation of new aspiration devices.

\section{Period-to-period data from frontline contact} aspiration strategies at the authors' institute The frontline CA-based EVT protocol has been applied at the authors' institute since April of 2009 when the Penumbra System first launched in Korea (Figure 4). As previously described, we only used a Penumbra reperfusion catheter for $C A$, rather than the whole Penumbra System. The CA technique used was FAST. ${ }^{17}$ FAST was repeatedly attempted to achieve reperfusion until November 2010 when the Solitaire stent (Medtronic) was commercially released. From that time, the authors applied a 'switching' concept from CA (FAST) to SR using the Solitaire when FAST was not effective after three attempts. ${ }^{27}$ This protocol was maintained with a few minor changes, such as using the Penumbra Max for CA and Trevo retriever (Stryker) for SR in some cases, until September of 2016 when the ACE 68 (Penumbra) and Sofia (MicroVention) were imported to Korea. As these new catheters have a larger internal diameter and more stable support, we changed the rescue EVT from the bare SR technology to CASPER when switching was required. As shown in Figure 4, procedure and patient outcomes improved over time, which may be connected to various factors including the accumulation of the neurointerventionalists' experiences (achieving higher "first-pass effect" and prompt switching to rescue EVT), more appropriate patient selection (due to advances in stroke imaging and protocols), and a better system of care (faster patient transfer and in-hospital triage).
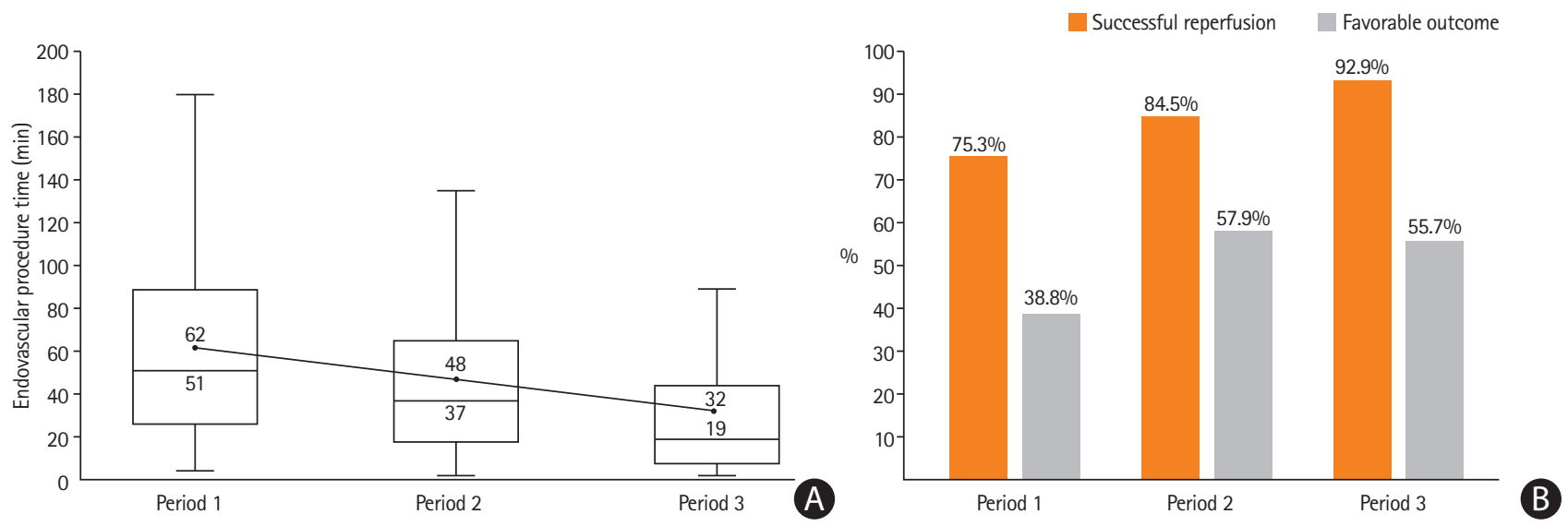

Figure 4. Period-to-period data from the endovascular registry of the authors' institute (unpublished data). We used a frontline contact aspiration based endovascular thrombectomy protocol from 2009. (A) Endovascular procedure times (minutes) decreased by period-to-period (upper value: mean; lower value: median). (B) The rate of successful reperfusion (modified Thrombolysis In Cerebral Infarction 2b/3) and favorable clinical outcome (modified Rankin Scale [mRS] 0-2 or equal to prestroke $m R S$ at 3 months) increased by period-to-period. Period 1: forced arterial suction thrombectomy (FAST) only (April 2009 to Novembet 2010; $n=85$ ). Period 2: FAST to stent retriever switching (December 2010 to September 2016; $n=489$ ). Period 3: FAST to CASPER (October 2016 to June 2018; $n=183$ ). 


\section{Special considerations for contact aspiration}

\section{Frontline contact aspiration for acute large vessel occlusion due to underlying intracranial atherosclerotic stenosis}

Acute LVO due to underlying ICAS, referred to as in situ thrombotic occlusion, is one of the major causes of failure or complicated EVT procedures. ${ }^{35}$ Such a procedural difficulty may lead to increased procedure duration and poor functional recovery. ${ }^{45}$ Because of its unique pathologic features, such as plaque rupture and re-thrombosis after primary reperfusion, patients with ICAS-related occlusions require a different EVT strategy from that used for patients with embolism-related stroke. The strategy comprises two components: (1) frontline thrombectomy to achieve primary recanalization of the target artery, and (2) rescue treatment to stabilize the irritable endothelium on the ICAS segment to prevent re-occlusion. ${ }^{46-48}$ As previously described, the two major EVT techniques currently considered as a frontline are SR and CA. However, it remains unclear which EVT technique is more effective in this variant of stroke due to a lack of studies. There have been only a few retrospective, single-arm case series regarding this topic to date. A case series reported that frontline CA was effective in $62.5 \%$ ( 25 of 40 ) of patients with an in situ thrombo-occlusion, and conversion to SR was additionally required for the remaining $37.5 \%$ of the cases. ${ }^{47}$ On the contrary, two other frontline SR-based studies for ICAS-related occlusions demonstrated that SR was effective in 88.9\% (eight of nine) and in 93.1\% (27 of 29) of patients as a frontline EVT. ${ }^{46,49}$ Based on the authors' experience, there are several possible benefits of the SR over CA in patients with ICAS-related occlusions. First, there can be a problem with contact during CA. In the case of proper contact achieved between the tip of the catheter and the thrombus, clot retrieval is relatively easy, as ICAS-related occlusions tend to have smaller clot burden than embolic occlusions (Figure 5A and B). However, most ICAS segments are tapered and irregularly shaped due to preexisting atherosclerosis within the vessel wall, making it difficult to place the tip of a large-bore aspiration catheter in contact with the proximal surface of a thrombus (Figure 5C). In contrast, the SR is deployed across the stenotic segment; thus, it becomes fully engaged with the entire length of the clot (Figure 5D). Second, this can provide another potential advantage in revealing the underlying culprit stenosis more readily after stent retrieval..$^{50}$ Therefore, it is possible to plan a subsequent rescue therapy earlier, which may be helpful in reducing procedure time and achieving quality reperfusion. Third, a temporary bypass can be achieved in most cases by placing the SR across the target arterial occlusion site. The temporary restoration of flow may cause thrombus reduction or dissolution by endogenous thrombolysis.

\section{Frontline contact aspiration in acute basilar artery occlusion}

It is well known that acute stroke caused by BA occlusion tends to have devastating effects on patients. The outcome and mortality associated with BA occlusions are worse than those associated with anterior circulation stroke. ${ }^{51-53} \mathrm{Al}-$ though EVT has been validated as the standard of care in patients with anterior circulation stroke, ${ }^{8}$ the effectiveness and safety of modern EVT remains uncertain for patients with acute BA occlusion. However, modern EVT methods, which mostly involve $\mathrm{CA}$ and $\mathrm{SR}$, have continuously attempted to improve the patients' recovery in this variant of stroke, de-
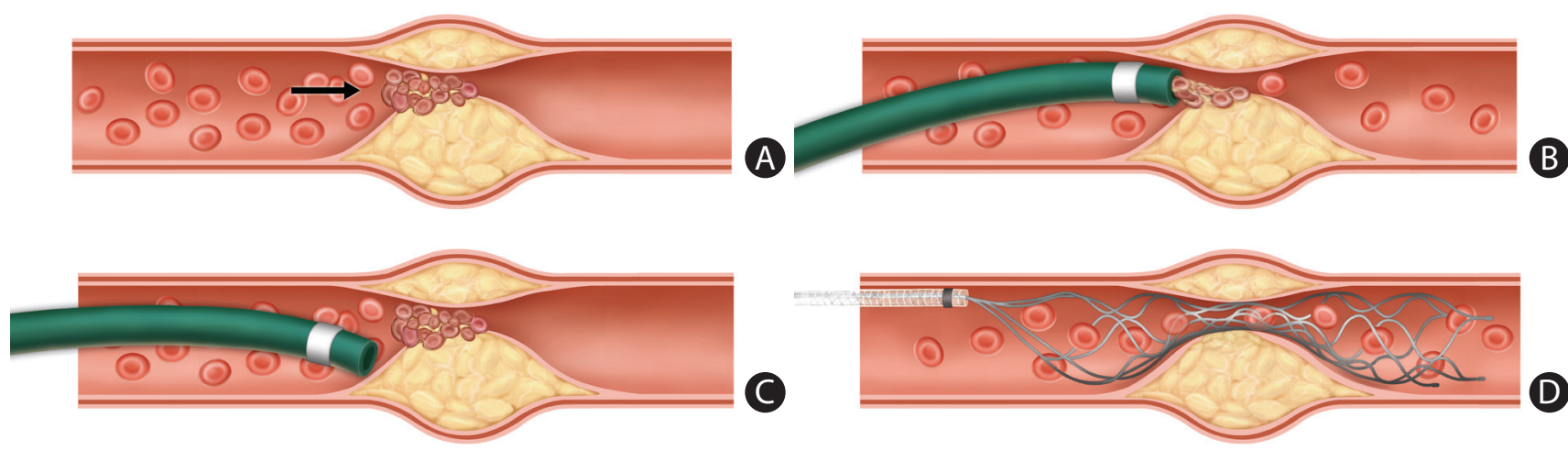

Figure 5. Frontline thrombectomy for acute large vessel occlusion (LVO) due to underlying intracranial atherosclerotic stenosis (ICAS). (A) In situ thrombosis is a main pathology of acute LVO due to underlying ICAS. Arrow denotes aggregated thrombi on the ruptured atherosclerotic plaque. (B) Clot retrieval is relatively easy when proper contact is achieved between the tip of a large-bore aspiration catheter and the proximal surface of a clot, because this type of occlusion generally has a small clot burden. (C) However, in some cases, contact is difficult because of the tapered and irregular anatomy of the stenotic lumen. (D) A stent retriever behaves according to a different mechanism, which is deployed across the stenotic segment and then becomes fully engaged through the entire length of a clot. 
spite limited results due to small sample sizes and heterogeneous patient populations..$^{54,55}$ In 2014, a case series reported superior performance of FAST, an early variant of CA, in patients with acute BA occlusion compared to intra-arterial fibrinolysis. ${ }^{56}$ In this study, the FAST group had a shorter procedure time (mean, 75.5 minutes vs. 113.3 minutes, $P=0.016$ ) and a higher successful revascularization rate ( $88 \%$ vs. $60 \%$, $P=0.017$ ) than the fibrinolysis group. Fair outcome, defined as mRS $0-3$, at 3 months was achieved in 34\% of patients undergoing FAST and $8 \%$ of patients undergoing fibrinolysis $(P=0.019)$. Notably, the authors postulated the potential benefit of CA in acute BA occlusions. For the FAST method, the aspiration catheter is advanced just proximal to the clot, then negative pressure is applied to aspirate the thrombus. In addition, the advancement of the aspiration catheter can be achieved without deep navigation distal to the clot assisted by the relatively straight anatomy of the BA. Given that the angiographically obscured distal artery beyond the BA occlusion is perforator-rich, distal deep navigation using a microwire and a microcatheter can have a risk of injuring the small arteries. Thus, the FAST method may reduce the chance of inadvertent hemorrhagic complications resulting from the injury of perforating arteries. More recently, there have been several reports demonstrating the superiority of CA over SR in acute BA occlusions..$^{54,57,58}$ Gory et al. ${ }^{57}$ analyzed procedural details for 100 patients with acute BA occlusion and reported that CA was superior to SR as a frontline strategy, which reported that $C A$ achieved a significantly higher rate of complete reperfusion (modified $\mathrm{TICl} 3,54.3 \%$ vs. $31.5 \%, P=0.021$ ) and a shorter procedure time (45 minutes vs. 56 minutes, $P=0.05$ ) than SR thrombectomy. However, they found similar rates of successful reperfusion (modified $\mathrm{TICl} 2 \mathrm{~b} / 3$ ) and good outcome between the two approaches. Similarly, two other case series reported that CA achieved a higher rate of complete recanalization and shorter procedure time than SR in patients with acute BA occlusion..$^{54,58}$ On the contrary, Mokin et al. ${ }^{59}$ found no significant differences in procedure time, rate of successful reperfusion, or rate of good outcomes between the SR and CA in a cohort of 100 patients with posterior circulation strokes. In a multi-center, retrospective observational study based on 212 patients with acute BA occlusion in Korea, SR and CA had similar outcomes as a frontline EVT in terms of successful reperfusion (modified $\mathrm{TICl} 2 \mathrm{~b} / 3,90.3 \%$ vs. $94 \%, P=0.371), m R S 0-2$ at 90 days ( $46.9 \%$ vs. $40.3 \%$, $P=0.369)$, hemorrhagic infarction ( $20.7 \%$ vs. $13.4 \%, P=0.205)$, and mortality (15.9\% vs. $16.4 \%, P=0.918){ }^{60}$ Thus, it remains controversial which method is a better frontline EVT in acute BA occlusions. However, with the recent improvements in procedural and patient outcomes, modern EVT might be considered as the standard of care for eligible patients with acute BA occlusion, and further RCTs are recommended to confirm the efficacy of modern EVT in the near future.

\section{Summary}

This article briefly reviewed the recent footprints of CA with a focus on related RCTs, technical skills, and device technologies. The initial results of CA were comparable to those of SR, allowing for a RCT (THERAPY) to compare CA plus intravenous alteplase vs intravenous alteplase alone. Although the THERAPY study failed to achieve the primary endpoint, the ASTER study recently showed a similar efficacy between $C A$ and $S R$ as a frontline EVT for successful recanalization among patients with acute LVO in the anterior circulation. Based on the review of the literature and the authors' experience, there are two key factors for improving results of CA: (1) technically, understanding practical skills, including troubleshooting steps to overcome difficulties during the delivery of a large-bore catheter, in order to achieve quick, quality reperfusion, and (2) conceptually, protocolizing the switch to rescue EVT (either SR or CASPER) when the recanalization is insufficient after a few attempts of CA. A frontline $\mathrm{CA}$ strategy means EVT is no longer solely dependent on $\mathrm{CA}$ alone, but rather the whole EVT procedure, including up to a couple of attempts of CA followed by prompt switching to rescue therapy. Thus, further RCTs investigating these factors are strongly recommended to confirm the best frontline EVT strategy considering efficacy, safety, and cost-effectiveness. In addition, device technologies including newer versions of aspiration catheters are continuously evolving and demonstrating noteworthy results; therefore, accumulation of experience and clinical data seems necessary to evaluate new aspiration devices for improved CA outcomes.

\section{Disclosure}

The authors have no financial conflicts of interest.

\section{References}

1. Berkhemer $O A$, Fransen $P S$, Beumer $D$, van den Berg LA, Lingsma $\mathrm{HF}_{1}$ Yoo $\mathrm{AJ}$, et al. $A$ randomized trial of intraarterial treatment for acute ischemic stroke. N Engl J Med 2015;372:11-20.

2. Goyal M, Demchuk AM, Menon BK, Eesa M, Rempel JL, Thornton J, et al. Randomized assessment of rapid endovascular treatment of ischemic stroke. N Engl J Med 2015;372:10191030. 
3. Campbell BC, Mitchell PJ, Kleinig TJ, Dewey HM, Churilov L, Yassi $\mathrm{N}$, et al. Endovascular therapy for ischemic stroke with perfusion-imaging selection. N Engl J Med 2015;372:10091018.

4. Jovin TG, Chamorro A, Cobo E, de Miquel MA, Molina CA, Rovira $A$, et al. Thrombectomy within 8 hours after symptom onset in ischemic stroke. N Engl J Med 2015;372:2296-2306.

5. Saver JL, Goyal M, Bonafe A, Diener HC, Levy El, Pereira VM, et al. Stent-retriever thrombectomy after intravenous t-PA vs. t-PA alone in stroke. N Engl J Med 2015;372:2285-2295.

6. Bracard S, Ducrocq X, Mas JL, Soudant M, Oppenheim C, Moulin $T$, et al. Mechanical thrombectomy after intravenous alteplase versus alteplase alone after stroke (THRACE): a randomised controlled trial. Lancet Neurol 2016;15:1138-1147.

7. Wahlgren N, Moreira T, Michel P, Steiner T, Jansen O, Cog-

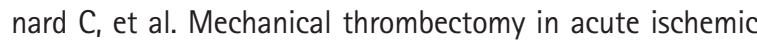
stroke: consensus statement by ESO-Karolinska Stroke Update 2014/2015, supported by ESO, ESMINT, ESNR and EAN. Int J Stroke 2016;11:134-147.

8. Powers WJ, Derdeyn CP, Biller J, Coffey CS, Hoh BL, Jauch EC, et al. 2015 American Heart Association/American Stroke Association focused update of the 2013 guidelines for the early management of patients with acute ischemic stroke regarding endovascular treatment: a guideline for healthcare professionals from the American Heart Association/American Stroke Association. Stroke 2015;46:3020-3035.

9. Hong KS, Ko SB, Yu KH, Jung C, Park SO, Kim BM, et al. Update of the Korean clinical practice guidelines for endovascular recanalization therapy in patients with acute ischemic stroke. J Stroke 2016;18:102-113.

10. Fargen KM, Arthur AS, Spiotta AM, Lena J, Chaudry I, Turner $\mathrm{RD}$, et al. A survey of neurointerventionalists on thrombectomy practices for emergent large vessel occlusions. J Neurointerv Surg 2017;9:142-146.

11. Lapergue B, Blanc R, Gory B, Labreuche J, Duhamel A, Marnat $G$, et al. Effect of endovascular contact aspiration vs stent retriever on revascularization in patients with acute ischemic stroke and large vessel occlusion: the ASTER randomized clinical trial. JAMA 2017;318:443-452.

12. Turk AS, Siddiqui AH, Mocco J. A comparison of direct aspiration versus stent retriever as a first approach ('COMPASS'): protocol. J Neurointerv Surg 2018;10:953-957.

13. Spiotta AM, Chaudry MI, Hui FK, Turner RD, Kellogg RT, Turk AS. Evolution of thrombectomy approaches and devices for acute stroke: a technical review. J Neurointerv Surg 2015; 7:2-7.

14. Starck EE, McDermott JC, Crummy AB, Turnipseed WD, Acher $\mathrm{CW}$, Burgess JH. Percutaneous aspiration thromboembolectomy. Radiology 1985;156:61-66.
15. Chapot R, Houdart E, Rogopoulos A, Mounayer C, Saint-Maurice JP, Merland JJ. Thromboaspiration in the basilar artery: report of two cases. AJNR Am J Neuroradiol 2002;23:282-284.

16. Imai $K$, Mori T, Izumoto $H$, Takabatake $N$, Kunieda T, Shimizu $\mathrm{H}$, et al. Clot removal therapy by aspiration and extraction for acute embolic carotid occlusion. AJNR Am J Neuroradiol 2006;27:1521-1527.

17. Kang DH, Hwang YH, Kim YS, Park J, Kwon O, Jung C. Direct thrombus retrieval using the reperfusion catheter of the penumbra system: forced-suction thrombectomy in acute ischemic stroke. AJNR Am J Neuroradiol 2011;32:283-287.

18. Turk AS, Spiotta A, Frei D, Mocco J, Baxter B, Fiorella D, et al. Initial clinical experience with the ADAPT technique: a direct aspiration first pass technique for stroke thrombectomy. J Neurointerv Surg 2014;6:231-237.

19. Hwang YH, Kang DH, Kim YW, Kim YS, Park SP, Suh CK. Outcome of forced-suction thrombectomy in acute intracranial internal carotid occlusion. J Neurointerv Surg 2013;5 Suppl $1:$ i81-i84.

20. Turk AS, Frei D, Fiorella D, Mocco J, Baxter B, Siddiqui $A$, et al. ADAPT FAST study: a direct aspiration first pass technique for acute stroke thrombectomy. J Neurointerv Surg 2014;6:260264.

21. Vargas J, Spiotta A, Fargen K, Turner R, Chaudry I, Turk A. Long term experience using the ADAPT technique for the treatment of acute ischemic stroke. J Neurointerv Surg 2017;9:437-441.

22. Delgado Almandoz JE, Kayan Y, Young ML, Fease JL, Scholz JM, Milner AM, et al. Comparison of clinical outcomes in patients with acute ischemic strokes treated with mechanical thrombectomy using either Solumbra or ADAPT techniques. $J$ Neurointerv Surg 2016;8:1123-1128.

23. Goyal M, Menon BK, van Zwam WH, Dippel DW, Mitchell PJ, Demchuk AM, et al. Endovascular thrombectomy after largevessel ischaemic stroke: a meta-analysis of individual patient data from five randomised trials. Lancet 2016;387:17231731.

24. Mocco J, Zaidat 00, von Kummer R, Yoo AJ, Gupta R, Lopes $D$, et al. Aspiration thrombectomy after intravenous alteplase versus intravenous alteplase alone. Stroke 2016;47:23312338.

25. Lapergue B, Blanc R, Guedin P, Decroix JP, Labreuche J, Preda $C_{1}$ et al. A direct aspiration, first pass technique (ADAPT) versus stent retrievers for acute stroke therapy: an observational comparative study. AJNR Am J Neuroradiol 2016;37:18601865.

26. Stapleton CJ, Leslie-Mazwi TM, Torok CM, Hakimelahi $R$, Hirsch JA, Yoo AJ, et al. A direct aspiration first-pass technique vs stentriever thrombectomy in emergent large vessel 
intracranial occlusions. J Neurosurg 2018;128:567-574.

27. Kang DH, Kim YW, Hwang YH, Park J, Hwang JH, Kim YS. Switching strategy for mechanical thrombectomy of acute large vessel occlusion in the anterior circulation. Stroke 2013;44:3577-2579.

28. Zaidat 00, Castonguay AC, Linfante I, Gupta R, Martin CO, Holloway WE, et al. First pass effect: a new measure for stroke thrombectomy devices. Stroke 2018;49:660-666.

29. Chueh JY, Kühn AL, Puri AS, Wilson SD, Wakhloo AK, Gounis MJ. Reduction in distal emboli with proximal flow control during mechanical thrombectomy: a quantitative in vitro study. Stroke 2013;44:1396-1401.

30. Jahan R. Solitaire flow-restoration device for treatment of acute ischemic stroke: safety and recanalization efficacy study in a swine vessel occlusion model. AJNR Am J Neuroradiol 2010;31:1938-1943.

31. Nguyen TN, Malisch T, Castonguay AC, Gupta $R$, Sun $\mathrm{CH}_{\text {, }}$ Martin $\mathrm{CO}$, et al. Balloon guide catheter improves revascularization and clinical outcomes with the solitaire device: analysis of the North American Solitaire Acute Stroke Registry. Stroke 2014;45:141-145.

32. Velasco A, Buerke B, Stracke CP, Berkemeyer S, Mosimann PJ, Schwindt $W$, et al. Comparison of a balloon guide catheter and a non-balloon guide catheter for mechanical thrombectomy. Radiology 2016;280:169-176.

33. Kang DH, Kim BM, Heo JH, Nam HS, Kim YD, Hwang YH, et al. Effect of balloon guide catheter utilization on contact aspiration thrombectomy. J Neurosurg 2018 Nov 1 [Epub]. https://doi.org/10.3171/2018.6.JNS181045.

34. Kang DH, Park J. Endovascular stroke therapy focused on stent retriever thrombectomy and direct clot aspiration: historical review and modern application. J Korean Neurosurg Soc 2017;60:335-347.

35. Kim BM. Causes and solutions of endovascular treatment failure. J Stroke 2017;19:131-142.

36. Yoo AJ, Andersson T. Thrombectomy in acute ischemic stroke: challenges to procedural success. J Stroke 2017;19:121-130.

37. Nikoubashman O, Wischer D, Hennemann HM, Büsen $M$, Brockmann C, Wiesmann M. Under pressure: comparison of aspiration techniques for endovascular mechanical thrombectomy. AJNR Am J Neuroradiol 2018;39:905-909.

38. Gross BA, Jadhav AP, Jovin TG, Jankowitz BT. Dump the pump: manual aspiration thrombectomy (MAT) with a syringe is technically effective, expeditious, and cost-efficient. J Neurointerv Surg 2018;10:354-357.

39. Froehler MT. Comparison of vacuum pressures and forces generated by different catheters and pumps for aspiration thrombectomy in acute ischemic stroke. Interv Neurol 2017;
6:199-206.

40. Nikoubashman O, Nikoubashman A, Büsen M, Wiesmann M. Necessary catheter diameters for mechanical thrombectomy with ADAPT. AJNR Am J Neuroradiol 2017;38:2277-2281.

41. Hu YC, Stiefel MF. Force and aspiration analysis of the ADAPT technique in acute ischemic stroke treatment. $J$ Neurointerv Surg 2016;8:244-246.

42. Möhlenbruch MA, Kabbasch $C_{1}$ Kowoll $A$, Broussalis $E_{1}$ Sonnberger $M$, Müller $M$, et al. Multicenter experience with the new SOFIA Plus catheter as a primary local aspiration catheter for acute stroke thrombectomy. J Neurointerv Surg 2017;9: 1223-1227.

43. Gory B, Turjman F. Contact aspiration with the new ARC catheter for thrombectomy of acute ischemic stroke: singlecenter results. World Neurosurg 2018;109:e374-e381.

44. Sallustio F, Pampana E, Davoli A, Merolla S, Koch G, Alemseged $F$, et al. Mechanical thrombectomy of acute ischemic stroke with a new intermediate aspiration catheter: preliminary results. J Neurointerv Surg 2018;10:975-977.

45. Kim YW, Hong JM, Park DG, Choi JW, Kang DH, Kim YS, et al. Effect of intracranial atherosclerotic disease on endovascular treatment for patients with acute vertebrobasilar occlusion. AJNR Am J Neuroradiol 2016;37:2072-2078.

46. Yoon W, Kim SK, Park MS, Kim BC, Kang HK. Endovascular treatment and the outcomes of atherosclerotic intracranial stenosis in patients with hyperacute stroke. Neurosurgery 2015;76:680-686.

47. Kang DH, Kim YW, Hwang YH, Park SP, Kim YS, Baik SK. Instant reocclusion following mechanical thrombectomy of in situ thromboocclusion and the role of low-dose intra-arterial tirofiban. Cerebrovasc Dis 2014;37:350-355.

48. Lee JS, Hong JM, Kim JS. Diagnostic and therapeutic strategies for acute intracranial atherosclerosis-related occlusions. J Stroke 2017;19:143-151.

49. Lee JS, Hong JM, Lee KS, Suh HI, Choi JW, Kim SY. Primary stent retrieval for acute intracranial large artery occlusion due to atherosclerotic disease. J Stroke 2016;18:96-101.

50. Baek JH, Kim BM, Kim DJ, Heo JH, Nam HS, Song D, et al. Importance of truncal-type occlusion in stentriever-based thrombectomy for acute stroke. Neurology 2016;87:15421550.

51. Schonewille WJ, Wijman CA, Michel P, Rueckert CM, Weimar C, Mattle HP, et al. Treatment and outcomes of acute basilar artery occlusion in the Basilar Artery International Cooperation Study (BASICS): a prospective registry study. Lancet Neurol 2009;8:724-730.

52. Mattle HP, Arnold M, Lindsberg PJ, Schonewille WJ, Schroth G. Basilar artery occlusion. Lancet Neurol 2011;10:1002- 
1014.

53. Singer OC, Berkefeld J, Nolte $\mathrm{CH}$, Bohner G, Haring HP, Trenkler $J$, et al. Mechanical recanalization in basilar artery occlusion: the ENDOSTROKE study. Ann Neurol 2015;77:415-424.

54. Son S, Choi DS, Oh MK, Hong J, Kim SK, Kang H, et al. Comparison of solitaire thrombectomy and Penumbra suction thrombectomy in patients with acute ischemic stroke caused by basilar artery occlusion. J Neurointerv Surg 2016;8:13-18.

55. Yoon W, Kim SK, Heo TW, Baek BH, Lee YY, Kang HK. Predictors of good outcome after stent-retriever thrombectomy in acute basilar artery occlusion. Stroke 2015;46:2972-2975.

56. Eom $\mathrm{Yl}$, Hwang $\mathrm{YH}$, Hong JM, Choi JW, Lim YC, Kang DH, et al. Forced arterial suction thrombectomy with the penumbra reperfusion catheter in acute basilar artery occlusion: a retrospective comparison study in 2 Korean university hospitals. AJNR Am J Neuroradiol 2014;35:2354-2359.

57. Gory B, Mazighi M, Blanc R, Labreuche J, Piotin M, Turjman
F, et al. Mechanical thrombectomy in basilar artery occlusion: influence of reperfusion on clinical outcome and impact of the first-line strategy (ADAPT vs stent retriever). $J$ Neurosurg 2018;129:1482-1491.

58. Gerber JC, Daubner D, Kaiser D, Engellandt K, Haedrich K,

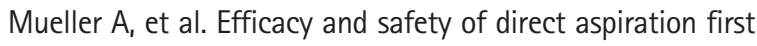
pass technique versus stent-retriever thrombectomy in acute basilar artery occlusion-a retrospective single center experience. Neuroradiology 2017;59:297-304.

59. Mokin M, Sonig A, Sivakanthan S, Ren Z, Elijovich L, Arthur $A$, et al. Clinical and procedural predictors of outcomes from the endovascular treatment of posterior circulation strokes. Stroke 2016:47:782-788.

60. Kang DH, Jung C, Yoon W, Kim SK, Baek BH, Kim JT, et al. Endovascular thrombectomy for acute basilar artery occlusion: a multicenter retrospective observational study. J Am Heart Assoc 2018;7:e009419. 